\title{
Subclinical subretinal fluid detectable only by optical coherence tomography in choroidal naevi-the SON study
}

\author{
Adrian T. Fung $\mathbb{1}^{1,2,3} \cdot$ Raymond Guan ${ }^{1}$ Veronica Forlani ${ }^{4} \cdot$ Yi-Chiao $\mathrm{Li}^{2,5} \cdot$ Jay Chhablani $\mathbb{1}^{6,7}$. \\ Dmitrii S. Maltsev $\mathbb{D}^{8} \cdot$ Dinah Zur $\mathbb{C}^{9} \cdot$ Matias Iglicki $\mathbb{D}^{10} \cdot$ Aude Couturier $^{11}$ - Ari Shinojima ${ }^{11,12}$.

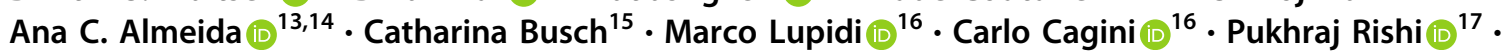 \\ Pierre-Henry Gabrielle $\mathbb{1}^{18} \cdot$ Samantha Fraser-Bell $\mathbb{1}^{2} \cdot$ Atchara Amphornphruet $^{19} \cdot$ Peranut Chotcomwongse $^{19}$. \\ Yan Hong Chen ${ }^{1} \cdot$ Marco Pellegrini ${ }^{4} \cdot$ For the International Retina Group
}

Received: 17 May 2020 / Revised: 16 September 2020 / Accepted: 21 September 2020 / Published online: 14 October 2020

(c) The Author(s), under exclusive licence to The Royal College of Ophthalmologists 2020

\begin{abstract}
Background Subretinal fluid is a risk factor for growth and malignant transformation of choroidal naevi, however it is unclear if this applies to subclinical fluid that is only detectable by optical coherence tomography (OCT). The objective of this study was to determine the prevalence and associations of subclinical but OCT-detectable subretinal fluid over choroidal naevi.

Methods Cross-sectional study of 309 consecutive cases of choroidal naevi imaged by OCT between July 2017 to January 2019. Multicentre international study involving ten retinal specialist centres. All patients presenting to retinal specialists had routine clinical examination and OCT imaging. The prevalence of subclinical OCT-detectable subretinal fluid over choroidal naevi and its associations with other features known to predict growth and malignant transformation were noted and analysed.

Results Of 309 identified consecutive cases, the mean patient age was 65 years, $89.3 \%$ of patients were Caucasian and $3.9 \%$ were Asian. The prevalence of subclinical but OCT-detectable subretinal fluid associated with choroidal naevi was $11.7 \%$ (36/309). Naevi with fluid were associated with larger basal diameters, greater thickness, presence of a halo, orange pigmentation, hyperautofluorescence, and hypodensity on B-scan ultrasonography.

Conclusion and relevance Of choroidal naevi where subretinal fluid is not visible on clinical examination, 11.7\% demonstrate subretinal fluid on OCT scans. These naevi more commonly exhibit features known to be associated with growth and transformation to melanoma. The presence of subclinical OCT-detectable fluid over choroidal naevi may assist in their risk stratification.
\end{abstract}

\section{Introduction}

Choroidal naevi are the most common intraocular tumours and are commonly found incidentally on fundus examination. They are mostly benign, asymptomatic, pigmented lesions with the majority located in the postequatorial fundus and can be found in up to $6.5 \%$ of the

Members of the International Retina Group are listed above references.

Adrian T. Fung

adrian.fung@sydney.edu.au

Extended author information available on the last page of the article
Caucasian population [1]. The risk of malignant transformation is low, $\sim 1$ in 4800 to 1 in 8845 naevi annually in Caucasians [2, 3]. Diagnosis of choroidal melanoma can be difficult as there are extensive overlapping features between naevi and melanoma. Features associated with choroidal naevi thought to be predictive of growth into melanoma include: greater thickness $>2 \mathrm{~mm}$ (on ultrasound), subretinal fluid (SRF), photopsia, symptomatic vision loss, orange pigment, hyperautofluorescence, margin touching or within $3 \mathrm{~mm}$ of the optic disc, tumour diameter $>5 \mathrm{~mm}$ on photography and naevus hollowness on ultrasound [4-7]. Early detection of malignancy is generally associated with a better prognosis for patients as it facilitates early intervention. The prognosis of choroidal melanoma is poor, with a 5-year mortality of $\sim 30 \%[8,9]$. 
In the past, studies assessing SRF around choroidal naevi were based purely on ophthalmoscopic clinical examination. Initially SRF was identified as a risk factor for growth of choroidal naevi, but over time this appears to have been interpreted as a risk factor for malignant transformation due to the strong association between growth and malignancy [6]. Since the introduction of optical coherence tomography (OCT), it has been possible to identify small volumes of SRF that are not visible clinically. A 2005 study by Shields et al. [10] detected clinically visible SRF in $16 \%$ and OCTdetectable SRF in $26 \%$ of a cohort of 120 naevi, suggesting that $10 \%$ of patients without SRF on clinical examination had OCT-detectable SRF. This study used time-domain (Zeiss Stratus) rather than spectral-domain (SD)-OCT and may therefore have underestimated the true prevalence of OCTdetectable SRF. A more recent study in 2019 by Shields et al. [7] demonstrated SD-OCT detectable SRF in $9 \%$ of choroidal naevi. Furthermore, the presence of such fluid at the apex and within $3 \mathrm{~mm}$ of the naevus demonstrated a statistically significant association with transformation into melanoma. However, this study did not exclude or report the number of patients with clinically detectable fluid. Other previous studies that have analysed SRF detectable by OCT in choroidal naevi also did not specifically exclude patients with clinically detectable SRF [7, 10-12].

To our knowledge there has been no study that has determined the prevalence of cases where SRF associated with choroidal naevi is only detectable by OCT and not identified by clinical examination. It therefore remains unclear if subclinical, 'OCT-detectable only' SRF confers the same risk for malignant transformation. In this paper we determine the prevalence of such cases and attempt to identify differences in other risk factors for progression to melanoma between naevi with and without OCTdetectable fluid.

\section{Materials and methods}

This was an international, multi-centre cross-sectional study of consecutive choroidal naevi seen between July 2017 and January 2019 seen across 10 retinal sites. Institutional review board (IRB) approval was obtained through the individual IRBs at the participating institutes. This research adhered to the tenets of the Declaration of Helsinki and informed consent was obtained by all patients.

\section{Inclusion and exclusion criteria}

All consecutive cases of clinically diagnosed choroidal naevi that could be adequately imaged with OCT were included. Both pigmented and amelanotic choroidal naevi were included. The diagnosis of a choroidal naevus was left to the discretion of the ophthalmologist, all of whom are trained in retinal and choroidal diseases. Cases were excluded if:

- There was clinically-detectable SRF (on slit-lamp or indirect ophthalmoscopy).

- There was concern the lesion might be a melanoma or other lesion. This included any lesions with a B-scan ultrasound measured apical thickness of over $3.0 \mathrm{~mm}$.

- A clear OCT scan could not be obtained (e.g., media opacity or if the lesion was too anterior).

- There was evidence of choroidal neovascularisation based on clinical examination (subretinal haemorrhage, hard exudate), OCT-angiography, or fluorescein angiography.

\section{Data collection}

For eligible patients, the following data were collected from the medical charts: age, ethnicity, laterality, visual acuity (converted from Snellen to LogMAR for statistical analysis) and presence or absence of symptoms. Clinical features of the choroidal naevi that were recorded included maximum basal diameter (measured from fundus photographs using digital callipers), the level of pigmentation (pigmented, partially pigmented, amelanotic), presence of a halo, presence of orange pigmentation and if lesion was $<3 \mathrm{~mm}$ from optic disc.

\section{Imaging}

Spectral-domain or swept-source OCT imaging of the choroidal naevi was performed with one of the following: Cirrus $^{\mathrm{TM}}$ HD-OCT 5000, Zeiss PLEX Elite 9000, Carl Zeiss Meditec AG, Jena, Germany; Spectralis HRA + OCT, Heidelberg Engineering GmbH, Heidelberg, Germany; Avanti, Optovue, Fremont, USA or 3D OCT-2000, Topcon, Tokyo, Japan. Horizontal and vertical rasters were performed through the lesion. The following were recorded on OCT: SRF maximal depth, SRF maximal horizontal diameter (on horizontal rasters), SRF maximal vertical diameter (on vertical rasters), choroidal naevus thickness (if measurable on enhanced depth-OCT or swept source-OCT), and the presence or absence of drusen. When the naevus was larger than the length of the OCT raster scans, attempts were made to scan the apex and base of the lesion to avoid missing SRF. Fundus autofluorescence (Visucam 524 Fundus imaging, Carl Zeiss Meditec, HRA + OCT, Heidelberg Engineering GmbH or California, Optos ${ }^{\oplus}$ PLC, Dunfermline, UK) was recorded of the naevus itself and the immediately surrounding fundus if this was abnormal. This was categorised as hyper/iso/hypo- or mixed autofluorescence according to the highest amount of 


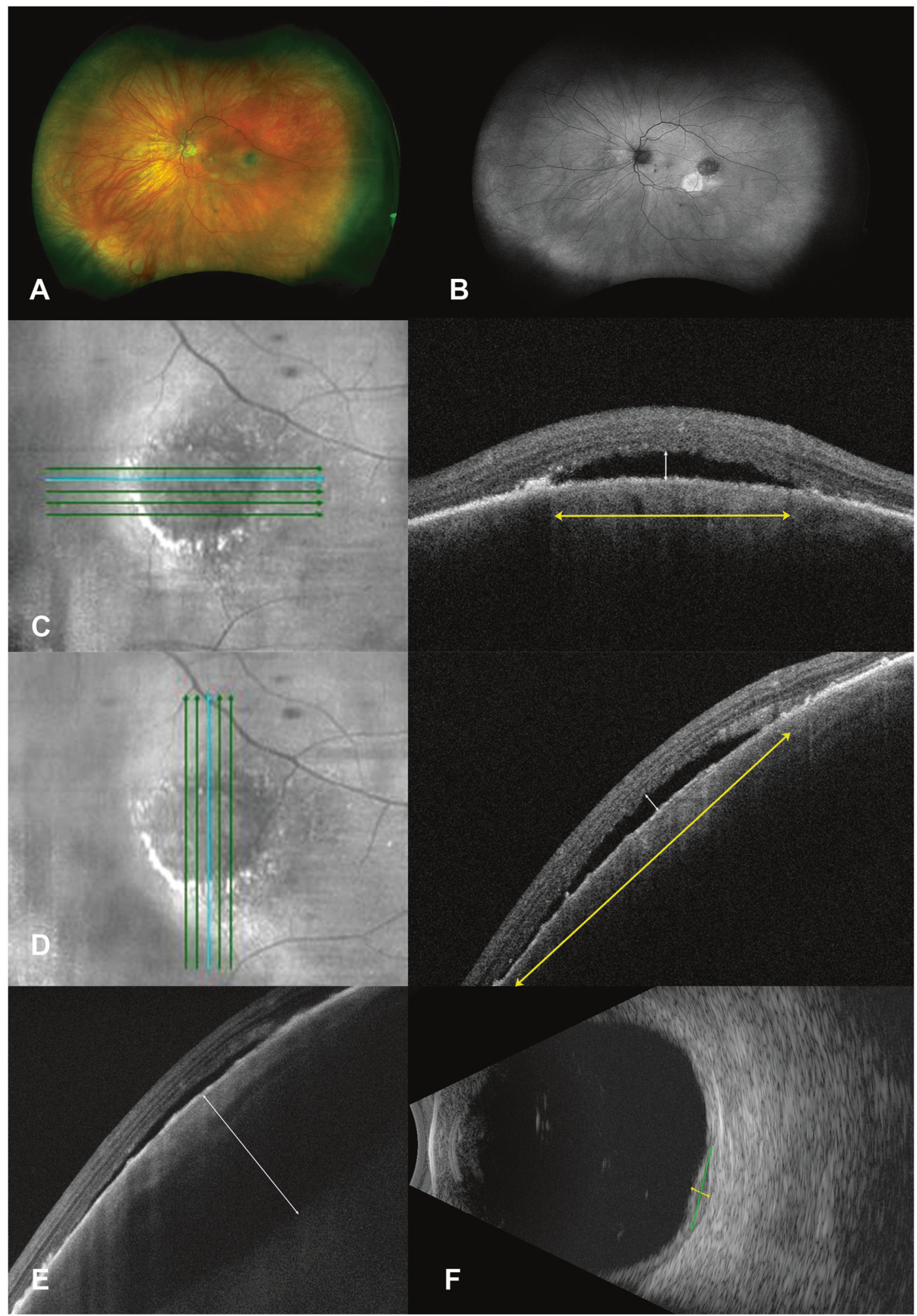

autofluorescence present. On B-scan ultrasonography (Eye Cubed, Ellex, Adelaide, Australia) the presence of SRF, echodensity and naevus thickness if measurable, were recorded. An example of a choroidal naevus included in the study and the multi-modal imaging analysis is shown in Fig. 1. 
Fig. 1 Multimodal imaging of a choroidal naevus. a Ultrawide field pseudocolour image (California, Optos ${ }^{\circledast}$ PLC, Dunfermline, UK) showing a pigmented choroidal naevus temporal to the macula. There is no clinical SRF, orange pigment or drusen. $\mathbf{b}$ There is a circular pool of hyperautofluorescence immediately inferonasal to the naevus. c Horizontal OCT rasters over the naevus were used to identify the maximal depth (white double ended arrow) and maximal horizontal diameter (yellow double-ended arrow) of SRF. d Vertical OCT rasters over the naevus were used to identify the maximal depth (white double ended arrow) and maximal vertical diameter (yellow double-ended arrow) of SRF. e Enhanced-depth imaging OCT outlines the posterior margin of the naevus for thickness calculation (double ended arrow). $\mathbf{f}$ B-scan ultrasonography shows the lesion to be hypodense and allows for further thickness calculation.

Prevalence was determined by calculating the percentage of choroidal naevi with OCT-detectable fluid. Choroidal naevi with and without OCT-detectable fluid were compared with regards to other risk factors known to be associated with a higher risk of malignant transformation. Statistical analysis was performed using IBM SPSS v23.0. Correlation between categorical variables were computed using Pearson's $\chi 2$ test while correlation between categorical and continuous variables were calculated using the Mann-Whitney $U$ test. Regarding continuous variables, in comparison of age, the assumptions of equal variance and normal distribution were valid, so T-test was used to compare the means. In the comparison of diameter and thickness on ultrasound, both assumptions were not valid, therefore the non-parametric Mann-Whitney U test was used to compare the means.

\section{Results}

In total, 309 consecutive cases of choroidal naevi were identified and scanned with OCT. The mean age was 65 years (range, 11-94 years, SD: 15.0). The majority of patients were Caucasian $(89 \%, 276 / 309)$. The mean logMAR (logarithm of the minimal angle of resolution) visual acuity was $0.08(20 / 24)$ (median, $0.00(20 / 20)$, range, -0.18 $(20 / 13)-2.00(\mathrm{CF}))$. Out of the 309 choroidal naevi, $11.7 \%$ (36/309) exhibited SRF only on OCT images and not ophthalmoscopy. No fluid was visible on B-scan in any case. Patient demographics are shown in Table 1 and history, examination, and imaging features of interest in Table 2.

The vast majority of naevi were asymptomatic regardless of fluid status. 4\% (12/309) of total cases exhibited symptoms, three of such cases had fluid on OCT $(8 \%, 3 / 36)$ versus nine cases of naevi without fluid $(3 \%, 9 / 273)$. The most commonly reported symptoms were decreased visual acuity $(33 \%, 4 / 12)$, blurred vision $(25 \%, 3 / 12)$, and headache $(17 \%, 2 / 12)$. Regarding subretinal fluid, the mean and ranges of the maximal depth, horizontal diameter, and vertical diameters were $98 \mu \mathrm{m} \quad(9-609 \mu \mathrm{m}), 1830$ $(133-5575 \mu \mathrm{m})$ and $1713 \mu \mathrm{m}(82-4776 \mu \mathrm{m})$ respectively.
Table 1 Prevalence of optical coherence tomography-detectable subretinal fluid associated with choroidal naevi demographics and features in 309 eyes of 309 patients.

\begin{tabular}{ll}
\hline Features & Patients $(n=309)$ \\
\hline Age, mean (median, range), years & $65(67,11-94)$ \\
Ethnicity & \\
$\quad$ White & $89.3 \%(276)$ \\
Asian & $3.9 \%(12)$ \\
$\quad$ Unknown & $6.8 \%(21)$ \\
Eye & \\
$\quad$ Right & $55.3 \%(171)$ \\
Left & $44.7 \%(138)$ \\
Visual Acuity mean (median, range), logMAR & $0.08(0.00,-0.18-2.00)$ \\
Subretinal Fluid on OCT & $11.7 \%(36)$ \\
$\quad$ Yes & $88.3 \%(273)$ \\
No & \\
\hline
\end{tabular}

Naevi with fluid were significantly larger (mean diameter $5.0 \mathrm{~mm}$, range $1.6-10.0 \mathrm{~mm}$ ) than those without fluid (mean $3.45 \mathrm{~mm}$, range $1.0-12.0 \mathrm{~mm})(p<0.001)$. Naevi with fluid were also thicker (mean $1.78 \mathrm{~mm}$, range, $0.0-2.8 \mathrm{~mm}$ on Bscan; mean $961 \mu \mathrm{m}$, range $266-1676 \mu \mathrm{m}$ on OCT) compared to those without fluid (mean $1.29 \mathrm{~mm}$, range, 0.0-2.9 mm on B-scan; mean $490 \mu \mathrm{m}$, range $130-2290 \mu \mathrm{m}$ on OCT) ( $p=0.002$ for B-scan, $p<0.001$ for OCT).

Orange pigmentation was more commonly found in naevi with fluid $(11 \%$ ) compared to those without $3 \%$ ( $p$ $=0.018$ ). A halo was present on clinical examination in $15 \%$ of naevi with fluid on OCT versus $3 \%$ without fluid $(p=0.001)$. Autofluorescence imaging was able to be captured in 266 eyes. Of these, naevi with subclinical OCT-detectable fluid were more likely to be hyperautofluorescent $(15 \%)$ compared to naevi without fluid $(4 \%)$ $(p=0.008)$. Of 258 patients in whom B-scan ultrasonography was performed, cross-tabulation of naevi showed those with OCT-detectable fluid were more likely to be hypodense compared to naevi without fluid ( $p=$ 0.001).

Pigmentation pattern, presence of drusen, and location in relation to the optic nerve was not statistically different in naevi with and without OCT-detectable fluid.

\section{Discussion}

Our study found that in $11.7 \%$ of cases of naevi where the clinician deemed there was no SRF, there was in fact subtle SRF visible on OCT alone. Naevi with fluid detectable on OCT were larger, thicker, more likely to demonstrate orange pigmentation, exhibit a halo, be hyperautofluorescent and demonstrate hypodensity on B-scan ultrasound. These factors, with the exception of halo presence, have previously been identified as risk factors for growth and malignant 
Table 2 Comparison of findings between naevi with and without subretinal fluid in 309 eyes of 309 patients.

\begin{tabular}{|c|c|c|c|}
\hline \multirow[t]{2}{*}{ Findings $^{\mathrm{a}}$} & \multicolumn{3}{|l|}{ Patients } \\
\hline & $\begin{array}{l}\text { Subretinal fluid } \\
(n=36)\end{array}$ & $\begin{array}{l}\text { No subretinal fluid } \\
(n=273)\end{array}$ & $P$ value \\
\hline Symptoms & & & $p=0.147$ \\
\hline Present $(n)$ & $8 \%(3)$ & $3 \%(9)$ & \\
\hline Absent $(n)$ & $92 \%(33)$ & $97 \%(263)$ & \\
\hline Diameter mean, (mm) (range) & $5.0(1.6-10.0)$ & $3.45(1.0-12.0)$ & $p<0.001$ \\
\hline $\begin{array}{l}\text { Thickness on B scan mean, } \\
(\mathrm{mm}) \text { (range) }\end{array}$ & $1.8(0.0-2.8)$ & $1.3(0.0-2.9)$ & $p=0.002$ \\
\hline $\begin{array}{l}\text { Thickness on OCT mean, } \\
(\mu \mathrm{m}) \text { (range) }\end{array}$ & $961(266-1676)$ & $490(130-2290)$ & $p<0.001$ \\
\hline Pigmentation & & & $p=0.753$ \\
\hline Pigmented $(n)$ & $86 \%(31)$ & $90 \%(245)$ & \\
\hline Partially Pigmented $(n)$ & $8 \%(3)$ & $8 \%(20)$ & \\
\hline Amelanotic $(n)$ & $6 \%(2)$ & $3 \%(7)$ & \\
\hline Orange Pigmentation & & & $p=0.018$ \\
\hline Present $(n)$ & $11 \%(4)$ & $3 \%(8)$ & \\
\hline Absent $(n)$ & $89 \%(32)$ & $97 \%(263)$ & \\
\hline Drusen & & & $p=0.638$ \\
\hline Present $(n)$ & $42 \%(14)$ & $38 \%(102)$ & \\
\hline Absent $(n)$ & $58 \%(19)$ & $62 \%(165)$ & \\
\hline Lesion $<3 \mathrm{~mm}$ from optic disc & & & $p=0.905$ \\
\hline Yes $(n)$ & $36 \%(13)$ & $37 \%(101)$ & \\
\hline No $(n)$ & $64 \%(23)$ & $63 \%(171)$ & \\
\hline Halo & & & $p=0.001$ \\
\hline Present $(n)$ & $15 \%(5)$ & $3 \%(8)$ & \\
\hline Absent $(n)$ & $85 \%(28)$ & $97 \%(264)$ & \\
\hline \multicolumn{4}{|l|}{ Autofluorescence } \\
\hline Hyper-autofluorescent $(n)$ & $15 \%(5)$ & $4 \%(9)$ & $p=0.008$ \\
\hline Iso-autofluorescent $(n)$ & $3 \%(1)$ & $57 \%(133)$ & \\
\hline Hypo-autofluorescent $(n)$ & $27 \%(9)$ & $17 \%(40)$ & \\
\hline Mixed & $56 \%(19)$ & $22 \%(50)$ & \\
\hline \multicolumn{4}{|l|}{ Echodensity } \\
\hline Hypodense $(n)$ & $14 \%(4)$ & $2 \%(5)$ & $p=0.001$ \\
\hline Isodense $(n)$ & $29 \%(8)$ & $61 \%(141)$ & \\
\hline Hyperdense $(n)$ & $57 \%(16)$ & $37 \%(84)$ & \\
\hline
\end{tabular}

${ }^{\mathrm{a}}$ Of patients with recordable data.

transformation [4]. Tumour size with respect to thickness and largest basal diameter has been shown to be the greatest risk factor for malignant transformation [13]. Hyperautofluorescence as an identifiable risk factor for growth could partially be attributable to the inherent appearance of subretinal fluid and orange pigmentation [14]. Although the findings of halo associated with OCT fluid was statistically significant, it was only present in a very small proportion of lesions and therefore is of questionable clinical significance. There was no significant difference in terms of cases of symptomatic patients, pigmentation, drusen absence, and lesion distance to the optic disc.

Previous studies have suggested that retinal changes could be identified to distinguish between 'active' and 'inactive' subretinal fluid. Photoreceptor morphology on the retina, such as 'shaggy photoreceptors' as described by Shields et al. [15]. may indicate the associated SRF is of active nature. Active
SRF was suggested to be associated with transformation to melanoma whilst inactive fluid has been attributed to chronic retinal pigment epithelial and retinal changes found over stable naevi [11]. However, a recent study by Yaghy et al. [16] demonstrated retinal changes caused by subretinal fluid associated with choroidal naevi followed a set longitudinal evolutionary course. Photoreceptor morphology was initially normal but transitioned to 'shaggy' then 'retracted' then absent the longer SRF was present. It was concluded that using photoreceptor morphology as an indicator for SRF chronicity had no correlation with naevus growth into melanoma [16]. As such, we did not assess photoreceptor morphology in this study.

The pathogenesis of subretinal fluid itself is unclear despite its clinical significance as a risk factor for malignant transformation. A recent study by $\mathrm{Yu}$ et al. [17] proposed that the presence of SRF was due to the mass effect of the nevus resulting in choriocapillaris compression, subsequent extravasation of SRF in combination with retinal pigment epithelium dropout leading to accumulation of subretinal fluid. It is suggested that SRF itself is not an independent risk factor for malignant transformation rather it is a sign of nevus growth itself. The true significance of subretinal fluid with respect to naevi and melanoma however has yet to be discerned and further research must be done.

This study has several limitations. The sample size is modest. As other retinal conditions were not excluded, visual acuity and symptoms could not necessarily be directly attributed to the naevi. The presence of SRF in itself is associated with hyperautofluorescence which in turn is associated with orange pigment, regardless of the risk of malignant transformation [14, 18]. All the investigators were retinal specialists, and the data may be affected by referral bias with lesions identified being larger or more concerning than those seen by optometrists or general ophthalmologists. There is inherent selection bias with the included choroidal naevi having to be posterior enough to obtain an adequate OCT scan. In studies by Shields and colleagues, $9 \%$ of naevi were found to be located anterior to the equator (equator to ora serrata) $[5,19]$. However no difference was found in rates of melanoma transformation between naevi located between the macula and equator versus anterior to the equator [5]. Histopathological confirmation that all the included tumours were naevi was not feasible due to the morbidity associated with biopsy, sampling error due to tumour heterogenicity and potential for tumour seeding of any choroidal melanoma [20]. Nevertheless, we feel confident that the cohort of patients represented naevi and not melanoma, based on the strict exclusion criteria. Lesions greater than $3.0 \mathrm{~mm}$ thickness on B-scan ultrasonography and indeterminate or concerning lesions for melanoma were excluded. In addition, the prevalence of choroidal naevi is much greater than melanoma. 
The mean age-adjusted incidence of uveal melanoma is 5.1 million with $90 \%$ of these cases being choroidal melanoma whereas the prevalence of choroidal naevi ranges from $1.4 \%$ to $6.5 \%$ depending on the population profile [21-23]. Finally, although we have been able to determine the prevalence of OCT-detectable SRF with respect to choroidal naevi, we do not know if this fluid is stable or changes with time. Further prospective studies are planned to track the choroidal naevi to determine if the volume of fluid changes, or if those with OCT-detectable SRF have a higher rate of conversion to melanoma than those without OCT-detectable SRF.

In summary, choroidal naevi demonstrate small amounts of SRF detectable by OCT in $11.7 \%$ of cases even when this is not visible on clinical examination. Naevi with this subclinical OCT-detectable fluid tend to be larger in diameter, thicker, have a halo, demonstrate orange pigment, exhibit hyperautofluorescence and be hypodense on B-scan ultrasonography. Most of these features are associated with naevus growth and malignant transformation. The objectivity and higher sensitivity of detecting SRF on OCT compared with clinical examination means that this imaging modality should be routinely used to stratify risk and monitor choroidal naevi for malignant transformation.

\section{Summary}

\section{What was known before}

Subretinal fluid is a risk factor for malignant transformation of naevi to melanoma, this fluid was previously detected clinically or on optical coherence tomography.

It is unclear whether this risk factor still applies for fluid that is undetectable on clinical examination and only visible on OCT.

\section{What this study adds}

Prevalence of subclinical subretinal fluid detectable only by optical coherence tomography associated with choroidal naevi.

Associations of subclinical OCT-detectable subretinal fluid with other features known to increase risk of malignant transformation.

International Retina Group Adrian T. Fung ${ }^{1,2,3}$, Jay Chhablani ${ }^{6,7}$, Dinah Zur ${ }^{9}$, Matias Iglicki ${ }^{10}$, Aude Couturier ${ }^{11}$, Catharina Busch ${ }^{14}$, Marco Lupidi ${ }^{15}$, Pierre-Henry Gabrielle ${ }^{17}$, Samantha Fraser-Bell ${ }^{2}$, Achara Ampornpruet ${ }^{18}$, Patricio J. Rodriguez-Valdez ${ }^{19}$, Zafer Cebeci $^{20}$, Ermete Giancipoli ${ }^{21,22}$, Voraporn Chaikitmongkol ${ }^{23}$, Mali Okada $^{24}$, Inês Lains ${ }^{25,26,27}$, Anna Sala-Puigdollers ${ }^{28}$, Malgorzata Ozimek $^{29}$, Matus Rehak ${ }^{14}$, Anat Loewenstein 9
${ }^{20}$ Instituto de Oftalmologia y Ciencias Visuales, Escuela de Medicina, Technologico de Monterrey, Monterrey, Mexico; ${ }^{21}$ Istanbul Faculty of Medicine, Department of Ophthalmology, Istanbul University, Istanbul, Turkey; ${ }^{22}$ Department of Surgical, Microsurgical and Medical Sciences, Eye Clinic, University of Sassari, Sassari, Italy; ${ }^{23}$ Department of Biomedical Sciences, Eye Clinic, University of Sassari, Sassari, Italy; ${ }^{24}$ Retina Division, Department of Ophthalmology, Faculty of Medicine, Chiang Mai University, Chiang Mai, Thailand; ${ }^{25}$ Royal Victorian Eye and Ear Hospital, Melbourne, VIC, Australia;

${ }^{26}$ Faculty of Medicine, University of Coimbra, Coimbra, Portugal;

${ }^{27}$ Association for Innovation and Biomedical Research on Light and Image, OCimbra, Portugal; ${ }^{28}$ Massachusetts Eye and Ear, Harvard Medical School, Boston, MA, USA; ${ }^{29}$ Institut Clinic d'Oftalmologia (ICOF), Hospital Clinic de Barcelona, Barcelona, Spain; ${ }^{30}$ Department of General Ophthalmology and Pediatric Ophthalmology Service, Medical University in Lublin, Lublin, Poland

\section{Compliance with ethical standards}

Conflict of interest The authors declare that they have no conflict of interest.

Publisher's note Springer Nature remains neutral with regard to jurisdictional claims in published maps and institutional affiliations.

\section{References}

1. Sumich P, Mitchell P, Wang JJ. Choroidal nevi in a white population: the blue mountains eye study. Arch Ophthalmol. 1998;116:645-50.

2. Ganley JP, Comstock GW. Benign nevi and malignant melanomas of the choroid. Am J Ophthalmol. 1973;76:19-25.

3. Singh AD, Kalyani P, Topham A. Estimating the risk of malignant transformation of a choroidal nevus. Ophthalmology 2005;112:1784-9.

4. Shields CL, Cater J, Shields JA, Singh AD, Santos MCM, Carvalho C. Combination of clinical factors predictive of growth of small choroidal melanocytic tumors. Arch Ophthalmol. 2000;118:360-4.

5. Shields CL, Furuta M, Berman EL, Zahler JD, Hoberman DM, Dinh $\mathrm{DH}$, et al. Choroidal nevus transformation into melanoma: analysis of 2514 consecutive cases. Arch Ophthalmol. 2009;127:981-7.

6. Shields CL, Shields JA, Kiratli H, De Potter P, Cater JR. Risk factors for growth and metastasis of small choroidal melanocytic lesions. Trans Am Ophthalmological Soc. 1995;93:259-75. discussion 75-79

7. Shields CL, Dalvin LA, Ancona-Lezama D, Yu MD, Di Nicola $\mathrm{M}$, Williams BK Jr, et al. Choroidal nevus imaging features in 3,806 cases and risk factors for transformation into melanoma in 2,355 cases: the 2020 Taylor R. Smith Victor T Curtin Lect RETINA. 2019;39:1840.

8. Kujala E, Makitie T, Kivela T. Very long-term prognosis of patients with malignant uveal melanoma. Invest Ophthalmol Vis Sci. 2003;44:4651-9.

9. Straatsma BR, Diener-West M, Caldwell R, Engstrom RE, Group* COMS. Mortality after deferral of treatment or no treatment for choroidal melanoma. J Ophthalmol. 2018;66:1395-400.

10. Shields CL, Mashayekhi A, Materin MA, Luo CK, Marr BP, Demirci $\mathrm{H}$, et al. Optical coherence tomography of choroidal nevus in 120 patients. Retina. 2005;25:243-52.

11. Espinoza G, Rosenblatt B, Harbour JW. Optical coherence tomography in the evaluation of retinal changes associated with 
suspicious choroidal melanocytic tumors. Am J Ophthalmol. 2004; 137:90-5.

12. Jonna G, Daniels AB. Enhanced depth imaging OCT of ultrasonographically flat choroidal nevi demonstrates 5 distinct patterns. Ophthalmol Retin. 2019;3:270-7.

13. Kaiserman I, Kaiserman N, Pe'er J. Long term ultrasonic follow up of choroidal naevi and their transformation to melanomas. Br J Ophthalmol. 2006;90:994-8.

14. Shields CL, Bianciotto C, Pirondini C, Materin MA, Harmon SA, Shields JA. Autofluorescence of choroidal melanoma in 51 cases. Br J Ophthalmol. 2008;92:617-22.

15. Shields CL, Kaliki S, Rojanaporn D, Ferenczy SR, Shields JA. Enhanced depth imaging optical coherence tomography of small choroidal melanoma: comparison with choroidal nevus. Arch Ophthalmol. 2012;130:850-6.

16. Yaghy A, Yu MD, Dalvin LA, Mazloumi M, Ferenczy SR, Shields CL. Photoreceptor morphology and correlation with subretinal fluid chronicity associated with choroidal nevus. Br J Ophthalmol. 2020;104:863-7

17. Yu MD, Dalvin LA, Ancona-Lezama D, Yaghy A, Ferenczy SR, Milman T, et al. Choriocapillaris compression correlates with choroidal nevus-associated subretinal fluid: OCT analysis of 3431 cases. Ophthalmology. 2020:127:863-7

18. Shields CL, Bianciotto C, Pirondini C, Materin MA, Harmon SA, Shields JA. Autofluorescence of orange pigment overlying small choroidal melanoma. Retina 2007;27:1107-11.

19. Shields CL, Furuta M, Thangappan A, Nagori S, Mashayekhi A, Lally $\mathrm{DR}$, et al. Metastasis of uveal melanoma millimeter-by-millimeter in 8033 consecutive eyes. Arch Ophthalmol. 2009;127:989-98.

20. Mashayekhi A, Shields CL, Eagle RC, Shields JA. Vitreous and preretinal seeding after transvitreal fine needle aspiration biopsy of choroidal melanoma. Retinal Cases Brief Rep. 2019.

21. Chien JL, Sioufi K, Surakiatchanukul T, Shields JA, Shields CL. Choroidal nevus: a review of prevalence, features, genetics, risks, and outcomes. [Review]. Curr Opin Ophthalmol. 2017;28:228-37.

22. Shields CL, Furuta M, Mashayekhi A, Berman EL, Zahler JD, Hoberman DM, et al. Clinical spectrum of choroidal nevi based on age at presentation in 3422 consecutive eyes. Ophthalmology 2008;115:546-52.e2.

23. Shields CL, Pirondini C, Bianciotto C, Materin MA, Harmon SA, Shields JA. Autofluorescence of choroidal nevus in 64 cases. Retina 2008;28:1035-43.

\section{Affiliations}

\section{Adrian T. Fung $\mathbb{1}^{1,2,3} \cdot$ Raymond Guan ${ }^{1} \cdot$ Veronica Forlani ${ }^{4} \cdot$ Yi-Chiao $\mathrm{Li}^{2,5} \cdot$ Jay Chhablani $\mathbb{1}^{6,7}$. Dmitrii S. Maltsev $\mathbb{( D D}^{8} \cdot$ Dinah Zur $\mathbb{D}^{9} \cdot$ Matias Iglicki $\mathbb{D}^{10}$ - Aude Couturier ${ }^{11}$ - Ari Shinojima ${ }^{11,12}$.

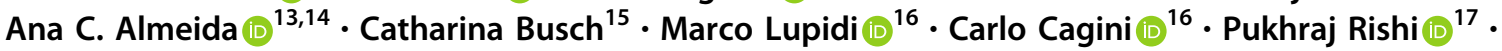 Pierre-Henry Gabrielle $\mathbb{D}^{18}$. Samantha Fraser-Bell $\mathbb{1}^{2}$. Atchara Amphornphruet ${ }^{19} \cdot$ Peranut Chotcomwongse $^{19}$. Yan Hong Chen ${ }^{1} \cdot$ Marco Pellegrini ${ }^{4} \cdot$ For the International Retina Group}

1 Westmead Clinical School, Discipline of Clinical Ophthalmology and Eye Health, University of Sydney, Sydney, NSW, Australia

2 Central Clinical School, Discipline of Clinical Ophthalmology and Eye Health, University of Sydney, Sydney, NSW, Australia

3 Department of Clinical Ophthalmology, Faculty of Medicine, Health and Human Sciences, Macquarie University, Sydney, NSW, Australia

4 Eye Clinic, Department of Biomedical and Clinical Science "Luigi Sacco", Luigi Sacco Hospital, University of Milan, Milan, Italy

5 Whitsunday Eye Surgery, Mackay, QLD, Australia

6 University of Pittsburgh, UPMC Eye Center, Pittsburgh, PA, USA

7 L.V. Prasad Eye Institute, Banjara Hills, Hyderabad, India

8 Department of Ophthalmology, Military Medical Academy, St. Petersburg, Russia

9 Ophthalmology Division, Tel Aviv Medical Center, affiliated to Sackler Faculty of Medicine, Tel Aviv University, Tel Aviv, Israel

10 Private Retina Service, University of Buenos Aires, Buenos Aires, Argentina
11 Department of Ophthalmology, Lariboisière Hospital, AP-HP, Universitè Paris 7- Sorbonne Paris Citè, Paris, France

12 Department of Ophthalmology, Keio University School of Medicine, Tokyo, Japan

13 Hospital Beatriz Ângelo, Lisbon, Portugal

14 Nova Medical School-Universidade Nova de Lisboa, Lisbon, Portugal

15 Department of Ophthalmology, University Hospital Leipzig, Leipzig, Germany

16 Department of Surgical and Biomedical Sciences, Section of Ophthalmology, University of Perugia, S. Maria della Misericordia Hospital, Perugia, Italy

17 Ocular Oncology \& Vitreoretina, Shri Bhagwan Mahavir Vitreoretinal Services, Sankara Nethralya, Chennai, Tamilnadu, India

18 Department of Ophthalmology, DIJON University Hospital, Burgundy, Dijon, France

19 Retina unit, Department of Ophthalmology, Rajavithi Hospital, Rangsit University, Bangkok, Thailand 